\title{
Congenital Anomalous Azygos Vein Drainage Causing Pulmonary Embolus in a 91-Year-Old Patient
}

\author{
Kai En Low ${ }^{1}$, Panduke Premathilake ${ }^{1}$, Lasanthi Pullaperuma ${ }^{1}$, Tammy Angel ${ }^{2}$ \\ ${ }^{1}$ Radiology Department, Luton and Dunstable Hospital, Luton, United Kingdom \\ ${ }^{2}$ Department of Elderly Medicine, Luton and Dunstable Hospital, Luton, United Kingdom
}

Received: 06/10/2021

Accepted: $14 / 10 / 2021$

Published: $10 / 11 / 2021$

How to cite this article: Low KE, Premathilake P, Pullaperuma L, Angel T. Congenital anomalous azygos vein drainage causing pulmonary embolus in a 91-year-old- patient. EJCRIM 2021;8: doi:10.12890/2021_002978.

Conflicts of Interests: The authors declare there are no competing interests.

Acknowledgements: We wish to acknowledge Dr Ching Wai Cheung who guided us on some of the haematological aspects of the clinical presentation. This article is licensed under a Commons Attribution Non-Commercial 4.0 License

\section{ABSTRACT}

Background: Retroaortic course and azygos continuation of aberrant left brachiocephalic vein is a rare venous anomaly, which is usually associated with congenital heart disease and pulmonary artery anomalies. Venous stasis is a cause of pulmonary arterial thromboembolism, which can result from venous anomalies.

Case presentation: We describe the case of a 91-year-old female admitted to our hospital with shortness of breath diagnosed with pulmonary embolism and infarctions by a CT pulmonary angiogram. CT also showed aberrant left brachiocephalic vein with vascular webs at its retroaortic course and azygos continuation, suggesting chronic venous thrombosis, which was considered to be the suspected source of emboli.

Conclusion: To our knowledge, this is the first report presenting this vascular anomaly manifesting with chronic venous thrombosis and pulmonary embolism. Although rare, awareness and identification of this entity is important, especially in the absence of obvious embolic sources or in patients with recurrent embolus/consolidation.

\section{LEARNING POINTS}

- Awareness of congenital anomalous drainage anatomy is important even if there has been a relatively benign clinical course.

- Alterations in morphology due to age and degenerative change may result in altered flow predisposing to chronic embolic phenomena.

- Recurrent consolidation may not necessarily be due to infection in the elderly, a recurrent embolic phenomenon may also be a cause, in this case without a common cause such as deep vein thrombosis.

\section{KEYWORDS}

Aortic anomalies, chronic thromboembolic disease, elderly patients

\section{INTRODUCTION}

In normal situs, the left brachiocephalic vein passes obliquely from left to right, anterior to the aortic arch and its major branches, to join the right brachiocephalic vein to form the SVC.

The retroaortic brachiocephalic vein with azygos continuation is relatively rare; approximately $0.2-1 \%$ of congenital cardiovascular anomalies ${ }^{[1,2]}$. Most of these were associated with other congenital cardiac anomalies such as aortic arch anomalies, tetralogy of Fallot, ASD, VSD and pulmonary trunk atresia. Multiple hypotheses were suggested to explain the embryogenesis of anomalous brachiocephalic vein with no clear consensus ${ }^{[3-5]}$. 
Usually, this anomaly has been discovered mainly in children and young adults during investigations for other associated cardiovascular anomalies. Presentation at older age with pulmonary embolism and infarctions due to the abnormal course leading to stagnation and thrombus formation has not been previously described.

\section{CASE PRESENTATION}

A 91-year-old female patient was admitted with sudden-onset shortness of breath. Examination demonstrated lung crackles with significant desaturation. The patient had multiple comorbidities including dementia, recent acute stroke, hypertension, acute renal failure and was delirious on admission.

The chest x-ray was unremarkable but D-dimer was raised (373 ng/ml). COVID-19 PCR testing on admission and multiple repeats were negative and there was no evidence of any other disease that could give rise to a hypercoagulable state. There was no clinical or imaging evidence of deep vein thrombosis.

An emergency CT pulmonary angiogram, with contrast through the left upper limb veins, revealed aberrant left brachiocephalic vein running inferiorly within the left mediastinum with a retroaortic course and an azygos continuation before draining into the superior vena cava. Vascular webs were seen within the retroaortic continuation in keeping with old thrombus formation. Small eccentric thrombi were seen within the lingula branch of the left main pulmonary artery and a segmental branch of the left lower lobe pulmonary artery. In the right main pulmonary artery, there was a pulmonary web. Wedge consolidation in the superior segment of the left lower lobe reflected possible acute pulmonary infarction or haemorrhage secondary to segmental pulmonary embolus.

The patient was anticoagulated but no further treatment was instituted due to age and the patient was managed expectantly. The patient passed away within 2 weeks in hospital from concurrent non-related comorbidities.

\section{DISCUSSION}

Isolated aberrant left brachiocephalic vein with a retroaortic course and azygos continuation are by themselves rare and are mostly asymptomatic with a benign clinical course. Complications mainly may be due to vascular compression and potential cardiothoracic surgical complications from unidentified aberrant anatomy.

In a previous case report, aortic coarctation secondary to the compressive effect of the brachiocephalic vein resulted in post-stenotic dilatation requiring surgical correction ${ }^{[6]}$.

In the current case report, altered flow due to kinking of the retroaortic brachiocephalic vein (Fig. 1), resulted in venous thrombosis in the region of altered flow (Fig. 2). The resultant thrombus traversed the brachiocephalic vein to the superior vena cava, right and left atria and resulted in pulmonary embolus (Fig. 3). The presence of webs suggests remodelling of previous thrombus in the aberrant vessel itself.

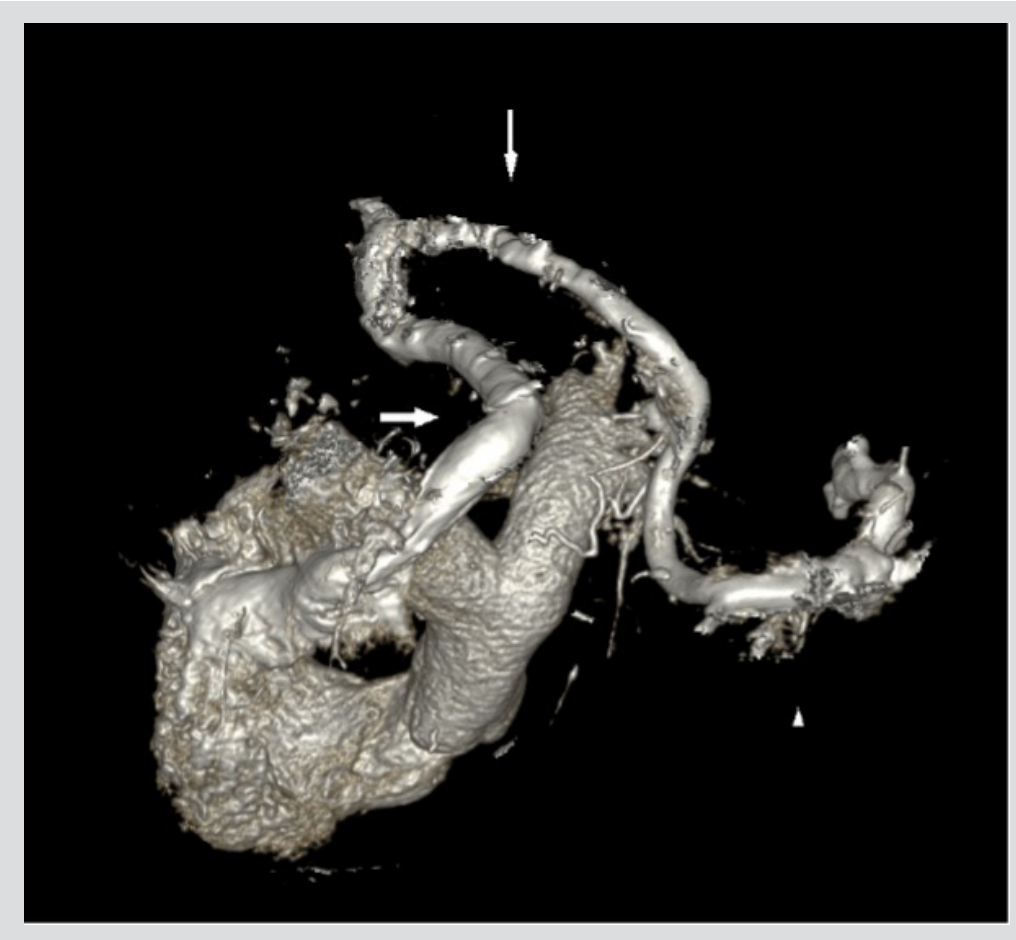

Figure 1. Volume-rendered image of the retroaortic left brachiocephalic vein, with the aorta removed to demonstrate the circuitous route. Triangle: left brachiocephalic vein; downward arrow: retroaortic portion; rightward arrow: azygos continuation to the superior vena cava 


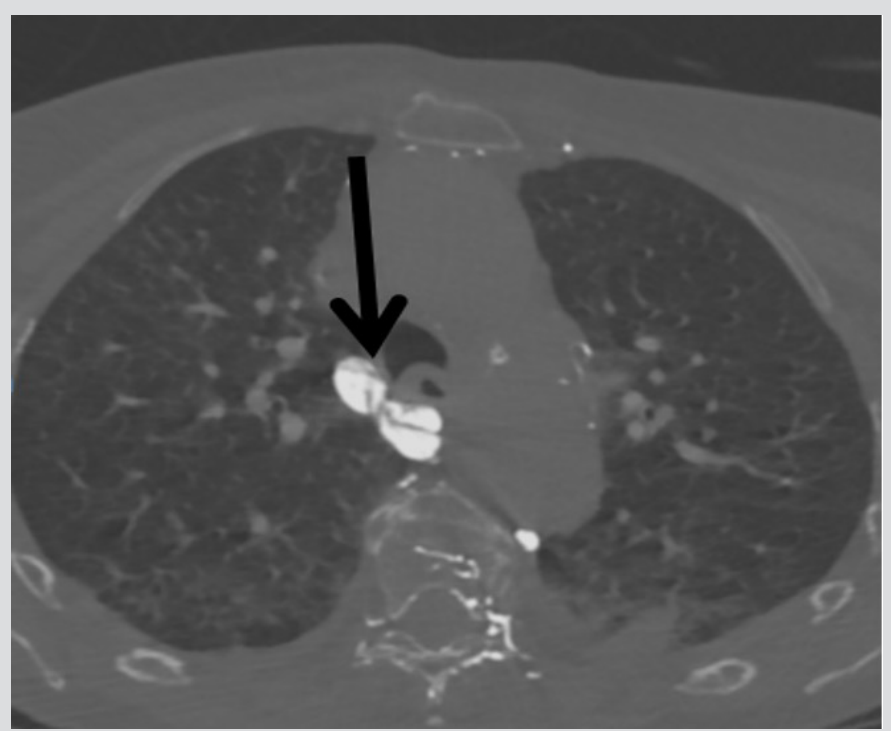

Figure 2. Pulmonary web in the retroaortic left brachiocephalic vein denoting chronic thrombus

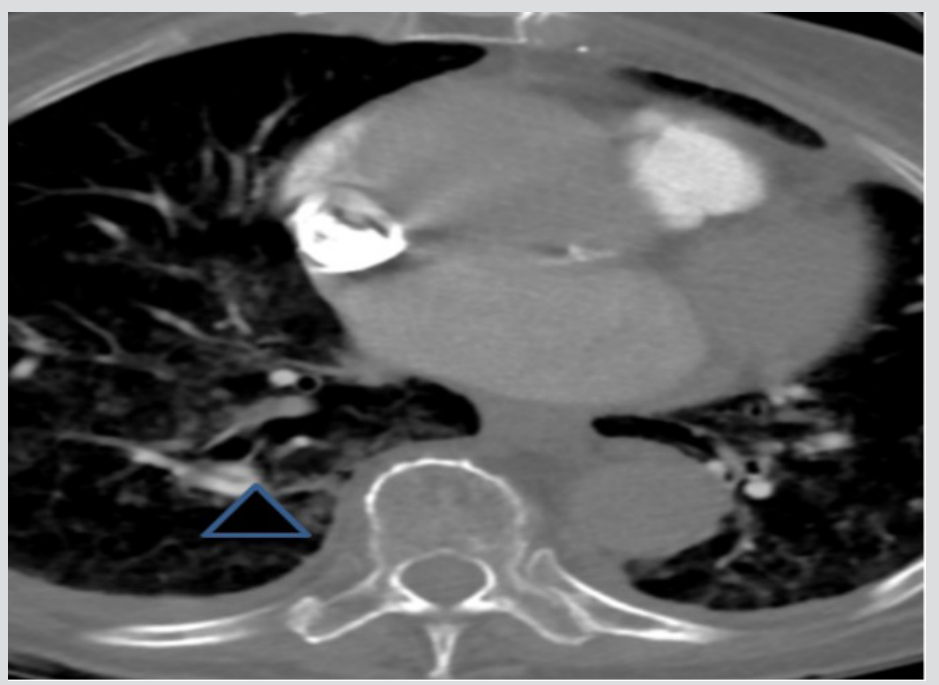

Figure 3. Chronic thrombus seen as a pulmonary web in the pulmonary arteries

This was seen just right after the kink resulting from the indentation of the ascending and descending aorta which was also enlarged, perhaps exacerbating the altered flow (Fig. 2). The presence of pulmonary webs in the right lower lobe pulmonary posterobasal segmental artery suggested chronic thromboembolic disease, together with the mosaic attenuation seen in the rest of the lungs (Fig. 4). The right ventricle was also dilated, suggesting chronic thromboembolic pulmonary hypertension (Fig. 5).

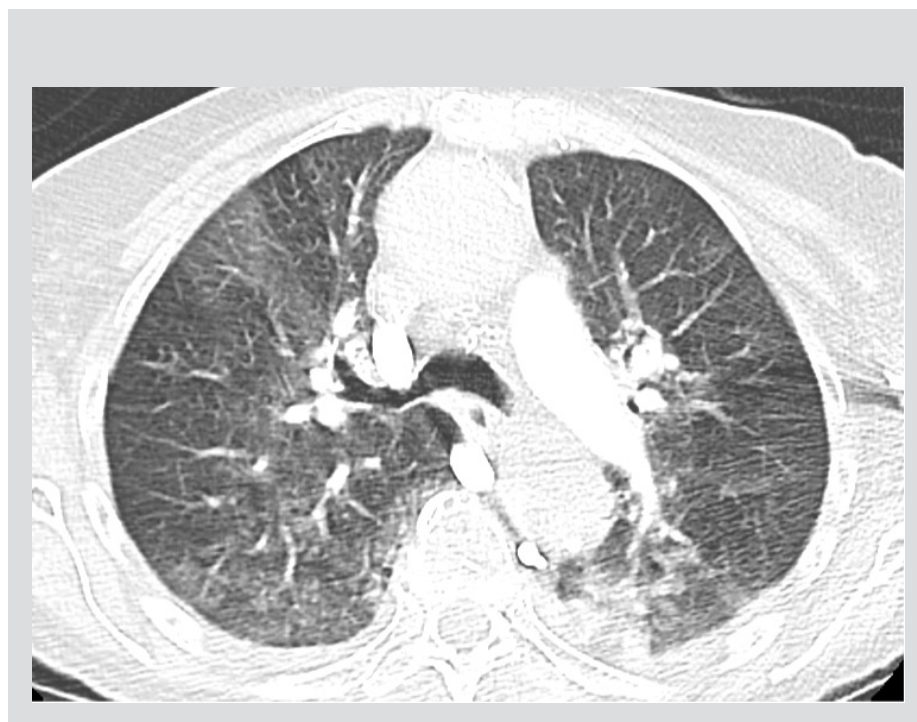

Figure 4. Mosaic attenuation in the lungs bilaterally suggesting chronic pulmonary hypertension. In addition, note the lower lobe consolidation

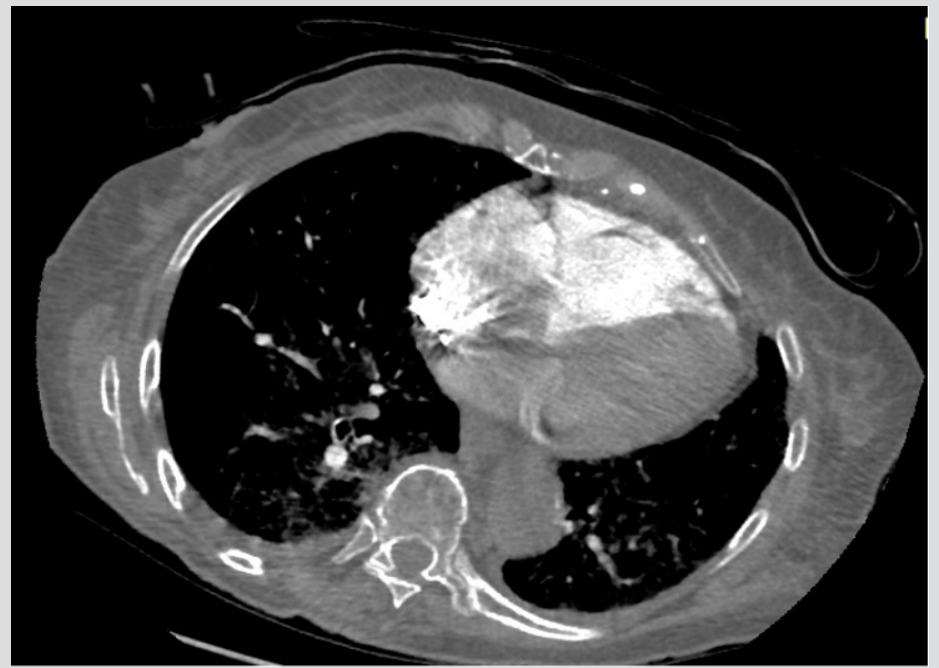

Figure 5. Right ventricular enlargement supporting chronic pulmonary hypertension

There was left lower lobar consolidation with a wedge shape, and the distal vessel demonstrated potential thrombus.

This raises important possibilities that in this patient there may have been multiple pulmonary embolus in the past, being misinterpreted as other causes of shortness of breath. In addition, patients with this vascular anomaly may be susceptible to morphological changes within the descending aorta, be it aneurysm or increased tortuosity which may then impinge on the vessel posteriorly.

This sequence of events has not been previously reported in the current literature. As the patient was relatively elderly, she was managed conservatively. 
The implication, therefore, is that such vascular anomalies must be carefully identified and classified on routine reporting. The radiologist should differentiate the descending part of an anomalous brachiocephalic vein from a persistent left superior vena cava ${ }^{[7]}$, an ascending vertical vein of a total anomalous pulmonary venous drainage ${ }^{[4,8]}$ and a left partial anomalous pulmonary venous drainage. It is also important not to misinterpret the retroaortic crossing segment of the anomalous vein as a pathologically enlarged lymph node in non-contrast $\mathrm{CT}^{[9]}$, as an elevated right pulmonary artery in patients with hypoplastic central pulmonary arteries or as an early branching right upper lobe pulmonary artery on cross-sectional echocardiography ${ }^{[7,10,11]}$.

\section{CONCLUSION}

Aberrant left brachiocephalic vein with a retroaortic course and an azygos continuation is rare and may be potentially associated with other congenital defects. In solitary cases the course may be benign over a long period, although there may be compressive effect either on the descending aorta (resulting in coarctation) or on the aberrant brachiocephalic vein either with direct compression or as a consequence of altered flow.

These patients are susceptible to aortic morphological changes which may result from degenerative changes, for example, aortic dilatation from hypertension or increased tortuosity.

There may also be chronic embolic disease arising from the aberrant brachiocephalic vein causing pulmonary hypertension.

Therefore, identification of the anomaly and its consequences is of the utmost importance. If identified on non-contrast imaging, a pulmonary angiogram is recommended with contrast in the left arm to opacify the aberrant vessel and to exclude filling defects.

\section{REFERENCES}

1. Azizova A, Onder O, Arslan S, Ardali S, Hazirolan T. Persistent left superior vena cava: clinical importance and differential diagnoses. Insights Imaging 2020;11:110.

2. Verma M, Pandey NN, Ojha V, Kumar S, Ramakrishnan S. Developmental anomalies of the superior vena cava and its tributaries: what the radiologist needs to know? Br J Radiol 2021;94:20200856.

3. Olry R, Lellouch A. The arterial system of the Japanese anatomist Buntaro Adachi. Hist Sci Med 2003;37(1):89-94

4. Minami M, Noda M, Kawauchi N, Shirouzu I, Nakajima J, Araki T, et al. Postaortic left innominate vein: radiological assessment and pathogenesis. Clin Radiol 1993;48(1):52-56.

5. Kim SH, Chung JW, Im JG, Choi YW, Choe YH, Han MC. Subaortic left innominate vein: radiologic findings and consideration of embryogenesis. $J$ Thorac Imaging 1999;14(2):142-146.

6. Ali İkidağ M. Retroaortic course and azygous continuation of an aberrant left brachiocephalic vein. Korean Circ J 2018;48:763-765.

7. Takada Y, Narimatsu A, Kohno A, Kawai C, Hara H, Harasawa A, et al. Anomalous left brachiocephalic vein: CT findings. J Comput Assist Tomogr 1992;16(6):893-896.

8. Gerlis LM, HoSY. Anomalous subaortic position of the brachiocephalic (innominate) vein: a review of published reports and report of three new cases. Heart 1989;61:540-545.

9. Chern MS, Shih-Chi Ko J, Tsai A, Wu MH, Teng MMH, Chang CY. Aberrant left brachiocephalic vein: CT imaging findings and embryologic correlation. Eur Radiol 1999;9(9):18351839.

10. Choi JY, Jung MJ, Kim YH, Noh Cl, Yun YS. Anomalous subaortic position of the brachiocephalic vein (innominate vein): an echocardiographic study. Heart 1990;64(6):385387.

11. Curtil A, Tronc F, Champsaur G, Bozio A, Sassolas F, Carret JP, et al. The left retro-aortic brachiocephalic vein: morphologic data and diagnostic ultrasound in 27 cases. Surg Radiol Anat 1999;21(4):251-254. 